\title{
Respiratory Review of 2013: Critical Care Medicine
}

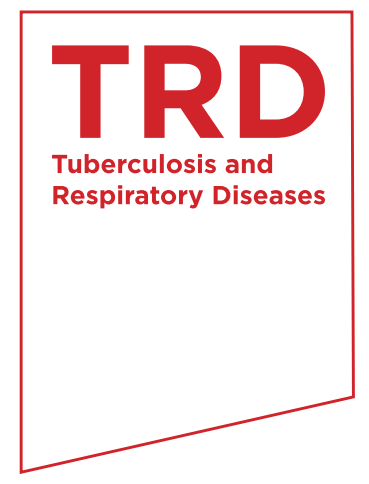

Hye Sook Choi, M.D., Ph.D.

Department of Internal Medicine, Dongguk University Gyeongju Hospital, Dongguk University College of Medicine, Gyeongju, Korea

Several papers on respiratory and critical care published from March 2012 to February 2013 were reviewed. From these, this study selected and summarized ten articles, in which the findings were notable, new, and interesting: effects of highfrequency oscillation ventilation on acute respiratory distress syndrome (ARDS); safety and efficacy of hydroxyethyl starch as a resuscitation fluid; long-term psychological impairments after ARDS; safety and efficacy of dexmedetomidine for sedation; B-type natriuretic peptide-guided fluid management during weaning from mechanical ventilation; adding of daily sedation interruptions to protocolized sedations for mechanical ventilation; unassisted tracheostomy collar of weaning from prolonged mechanical ventilations; and effects of nighttime intensivist staffing on the hospital mortality rates.

Keywords: Critical Care; High-Frequency Ventilation; Sepsis; Ventilator Weaning

\section{Introduction}

Critical care for the patients with sepsis, acute respiratory distress syndrome (ARDS), or mechanical ventilation in an intensive care unit (ICU) is improving but there are no promising medications for the treatment of these conditions. Many articles on critical care have been published over the past year. Original articles on respiratory and critical care published in clinical and scientific journals with an impact factor of 10 or more from March 2012 to February 2013 were reviewed. Among them, ten articles that provided notable and interesting results for the management of sepsis, ARDS, and mechani-

Address for correspondence: Hye Sook Choi, M.D., Ph.D.

Department of Internal Medicine, Dongguk University Gyeongju

Hospital, Dongguk University College of Medicine, 87 Dongdae-ro, Gyeongju 780-350, Korea

Phone: 82-54-770-8586, Fax: 82-54-770-8378

E-mail: maxymus72@hanmail.net

Received: May 11, 2013

Revised: May 16, 2013

Accepted: May 21, 2013

(9) It is identical to the Creative Commons Attribution Non-Commercial License (http://creativecommons.org/licenses/by-nc/3.0/).

Copyright ( $) 2013$

The Korean Academy of Tuberculosis and Respiratory Diseases.

All rights reserved. cal ventilation were chosen and summarized.

\section{High-Frequency Oscillation Ventilation (HFOV)}

1. The oscillation for ARDS treated early (OSCILLATE) trial

High-frequency oscillation in early acute respiratory distress syndrome. Fersguson et al. ${ }^{1} \mathrm{~N}$ Engl J Med 2013;368:795-805

BACKGROUND Previous trials suggesting that high-frequency oscillatory ventilation (HFOV) reduced mortality among adults with the acute respiratory distress syndrome (ARDS) were limited by the use of outdated comparator ventilation strategies and small sample sizes.

METHODS In a multicenter, randomized, controlled trial conducted at 39 intensive care units in five countries, we randomly assigned adults with new-onset, moderate-to-severe ARDS to HFOV targeting lung recruitment or to a control ventilation strategy targeting lung recruitment with the use of low tidal volumes and high positive end-expiratory pressure. The primary outcome was the rate of in-hospital death from any cause.

RESULTS On the recommendation of the data monitoring committee, we stopped the trial after 548 of a planned 1200 
patients had undergone randomization. The two study groups were well matched at baseline. The HFOV group underwent HFOV for a median of 3 days (interquartile range, 2 to 8 ); in addition, 34 of 273 patients (12\%) in the control group received HFOV for refractory hypoxemia. In-hospital mortality was $47 \%$ in the HFOV group, as compared with $35 \%$ in the control group (relative risk of death with HFOV, 1.33; 95\% confidence interval, 1.09 to 1.64; $\mathrm{P}=0.005$ ). This finding was independent of baseline abnormalities in oxygenation or respiratory compliance. Patients in the HFOV group received higher doses of midazolam than did patients in the control group (199 mg per day [interquartile range, 100 to 382] vs. $141 \mathrm{mg}$ per day [interquartile range, 68 to 240 ], $\mathrm{P}<0.001$ ), and more patients in the HFOV group than in the control group received neuromuscular blockers $(83 \%$ vs. $68 \%, \mathrm{P}<0.001)$. In addition, more patients in the HFOV group received vasoactive drugs ( $91 \%$ vs. $84 \%, \mathrm{P}=0.01$ ) and received them for a longer period than did patients in the control group ( 5 days vs. 3 days, $\mathrm{P}=0.01$ ).

CONCLUSIONS In adults with moderate-to-severe ARDS, early application of HFOV, as compared with a ventilation strategy of low tidal volume and high positive end-expiratory pressure, does not reduce, and may increase, in-hospital mortality.

1) Comments: This trial was terminated early for three reasons: on the basis of a consistent increased mortality observed with HFOV in three consecutive analyses; the increased need for vasoactive drugs in the HFOV group suggests a mechanism of harm that is not offset by better oxygenation and lung recruitment; and the effect size is sufficiently large. The authors suggest that three plausible mechanisms may contribute to increased mortality with HFOV: 1) higher mean airway pressures, 2) increased use of vasodilators, and 3) increased barotrauma. This study demonstrates harm caused by HFOV in early ARDS even when applied in patients with refractory hypoxemia.

\section{The oscillation in ARDS (OSCAR) study}

\section{High-frequency oscillation for acute respiratory distress syndrome. Young et al. ${ }^{2} \mathrm{~N}$ Engl J Med 2013; 368:806-13}

Methods In a multicenter study, we randomly assigned adults requiring mechanical ventilation for ARDS to undergo either HFOV with a Novalung R100 ventilator (Metran) or usual ventilatory care. All the patients had a ratio of the partial pressure of arterial oxygen $(\mathrm{PaO} 2)$ to the fraction of inspired oxygen $(\mathrm{FiO} 2)$ of $200 \mathrm{~mm} \mathrm{Hg}(26.7 \mathrm{kPa})$ or less and an expected duration of ventilation of at least 2 days. The primary outcome was allcause mortality 30 days after randomization.

Results There was no significant between-group difference in the primary outcome, which occurred in 166 of 398 patients (41.7\%) in the HFOV group and 163 of 397 patients (41.1\%) in the conventional-ventilation group $(\mathrm{P}=0.85$ by the chi-square test). After adjustment for study center, sex, score on the Acute Physiology and Chronic Health Evaluation (APACHE) II, and the initial PaO2:FiO2 ratio, the odds ratio for survival in the conventional-ventilation group was 1.03 (95\% confidence interval, 0.75 to $1.40 ; \mathrm{P}=0.87$ by logistic regression).

Conclusions The use of HFOV had no significant effect on 30day mortality in patients undergoing mechanical ventilation for ARDS.

1) Comments: The OSCAR trial did not show any benefit or harm resulting from the use of HFOV in ARDS patients. After adding this result to the meta-analysis ${ }^{3}$, which presents a reduced risk of death in HFOV compared with conventional ventilation, the estimated risk ratio from the pooled studies was modified to 0.90 (95\% confidence interval [CI], 0.76-1.07) $)^{2}$, indicating no significant benefit for HFOV. Why is there a difference in mortality between the OSCAR trial and the OSCILLATE trial ${ }^{\mathrm{l}}$ ? The OSCILLATE trial used the SensorMedics 3100B ventilator (CareFusion) for HFOV, which normally oscillates with an inspiratory:expiratory time ratio of 1:2, and the highest frequency. This approach led to a relatively higher mean airway pressure than the OSCAR trial. Therefore, it might be possible that the HFOV strategy employed in the OSCILLATE trial caused more harm. On the other hand, the control group strategies, which used the initial recruitment maneuver (the same as that used for the HFOV group) and a higher positive end-expiratory pressure protocol, appear to be more effective in the treatment of ARDS than those of the OSCAR trial. Based on the results of both trials ${ }^{1,2}$, I do not recommend the routine use of HFOV, but an adherence to lowtidal volume mechanical ventilation as a first-line treatment for ARDS.

\section{ARDS Outcomes}

\section{The ARDS cognitive outcomes study (ACOS)}

The adult respiratory distress syndrome cognitive outcomes study: long-term neuropsychological function in survivors of acute lung injury. Mikkelsen et al. ${ }^{4}$ Am J Respir Crit Care Med 2012;185:1307-15

Rationale: Cognitive and psychiatric morbidity is common and potentially modifiable after acute lung injury (ALI). However, practical measures of neuropsychological function for use in multicenter trials are lacking.

Objectives: To determine whether a validated telephonebased neuropsychological test battery is feasible in a multicenter trial. To determine the frequency and risk factors for long-term neuropsychological impairment.

Methods: As an adjunct study to the Acute Respiratory Dis- 
tress Syndrome Clinical Trials Network Fluid and Catheter Treatment Trial, we assessed neuropsychological function at 2 and 12 months post-hospital discharge.

Measurements and Main Results: Of 406 eligible survivors, we approached 261 to participate and 213 consented. We tested 122 subjects at least once, including 102 subjects at 12 months. Memory, verbal fluency, and executive function were impaired in 13\% (12 of 92), 16\% (15 of 96), and 49\% (37 of 76) of long-term survivors. Long-term cognitive impairment was present in 41 of the 75 (55\%) survivors who completed cognitive testing. Depression, post-traumatic stress disorder, or anxiety was present in $36 \%$ (37 of 102), 39\% ( 40 of 102), and 62\% (63 of 102) of long-term survivors. Enrollment in a conservative fluidmanagement strategy $(\mathrm{P}=0.005)$ was associated with cognitive impairment and lower partial pressure of arterial oxygen during the trial was associated with cognitive $(\mathrm{P}=0.02)$ and psychiatric impairment $(\mathrm{P}=0.02)$

Conclusions: Neuropsychological function can be assessed by telephone in a multicenter trial. Long-term neuropsychological impairment is common in survivors of ALI. Hypoxemia is a risk factor for long-term neuropsychological impairment. Fluid management strategy is a potential risk factor for longtermcognitive impairment.

1) Comments: The limitations of the study are 1) a small sample size, 2) only survivors who could be reached on the phone, and 3) a telephone-based survey. Nevertheless, the results show that most survivors of ARDS experienced longterm cognitive and psychiatric morbidity, particularly in the conservative fluid management group and in the patients with lower $\mathrm{PaO}_{2}$. Conservative fluid therapy improves the shortterm outcomes in $\mathrm{ARDS}^{5}$; however, we need to consider longterm brain health, and confirmatory studies may be required.

\section{Sedation for Mechanical Ventilation}

1. Daily sedation interruption in mechanically ventilated critically ill patients cared for with a sedation protocol: a randomized controlled trial. Mehta et al. ${ }^{6}$ JAMA 2012; 308:1985-92

Context Protocolized sedation and daily sedation interruption are 2 strategies to minimize sedation and reduce the duration of mechanical ventilation and intensive care unit (ICU) stay. We hypothesized that combining these strategies would augment the benefits.

Objective To compare protocolized sedation with protocolized sedation plus daily sedation interruption in critically ill patients. Design, Setting, and Patients Randomized controlled trial of 430 critically ill, mechanically ventilated adults conducted in 16 tertiary care medical and surgical ICUs in Canada and the United States between January 2008 and July 2011.
Intervention Continuous opioid and/or benzodiazepine infusions and random allocation to protocolized sedation ( $\mathrm{n}=$ 209) (control) or to protocolized sedation plus daily sedation interruption $(\mathrm{n}=214)$. Using validated scales, nurses titrated infusions to achieve light sedation. For patients receiving daily interruption, nurses resumed infusions, if indicated, at half of previous doses. Patients were assessed for delirium and for readiness for unassisted breathing.

Main Outcome Measure Time to successful extubation. Secondary outcomes included duration of stay, doses of sedatives and opioids, unintentional device removal, delirium, and nurse and respiratory therapist clinical workload (on a 10-point visual analog scale [VAS]).

Results Median time to successful extubation was 7 days in both the interruption and control groups (median [IQR], 7 [413 ] vs 7 [3-12]; interruption group hazard ratio, 1.08; 95\% CI, $0.86-1.35 ; P=.52$ ). Duration of ICU stay (median [IQR], 10 [517] days vs 10 [6-20] days; $P=.36$ ) and hospital stay (median [IQR], 20 [10-36] days vs 20 [10-48] days; $P=.42$ ) did not differ between the daily interruption and control groups, respectively. Daily interruption was associated with higher mean daily doses of midazolam (102 mg/d vs $82 \mathrm{mg} / \mathrm{d} ; P=.04$ ) and fentanyl (median [IQR], 550 [50-1850] vs 260 [0-1400]; $P<.001)$ and more daily boluses of benzodiazepines (mean, 0.253 vs $0.177 ; P=$ .007 ) and opiates (mean, 2.18 vs $1.79 ; P<.001)$. Unintentional endotracheal tube removal occurred in 10 of 214 (4.7\%) vs 12 of 207 patients $(5.8 \%)$ in the interruption and control groups, respectively (relative risk, $0.82 ; 95 \% \mathrm{CI}, 0.36-1.84 ; P=.64$ ). Rates of delirium were not significantly different between groups $(53.3 \%$ vs $54.1 \%$; relative risk, $0.98 ; 95 \% \mathrm{CI}, 0.82-1.17 ; P=.83$ ). Nurse workload was greater in the interruption group (VAS score, 4.22 vs 3.80; mean difference, 0.41 ; 95\% CI, 0.17-0.66; $P=.001$ ).

Conclusion For mechanically ventilated adults managed with protocolized sedation, the addition of daily sedation interruption did not reduce the duration of mechanical ventilation or ICU stay.

1) Comments: An earlier trial ${ }^{7}$ reported that daily sedation interruption makes ventilator duration shorter than 'usual care sedation' without a sedation protocol does. This study, however, compared a sedation strategy of daily sedation interruption plus protocolized sedation with a control group strategy of protocolized sedation. A nurse-implemented well-protocolized sedation that targets light sedation is more pragmatic than and superior to the usual care of the earlier trial design. Conclusively, adding daily sedation interruption to protocolized sedation does not have additional benefit. Rather, it increases the nurses' workload and the use of both sedatives and analgesics without shortening the time to extubation. 


\section{The MIDEX and PRODEX trials}

Dexmedetomidine vs midazolam or propofol for sedation during prolonged mechanical ventilation: two randomized controlled trials. Jakob et al. ${ }^{8}$ JAMA 2012;307:1151-60

Context Long-term sedation with midazolam or propofol in intensive care units (ICUs) has serious adverse effects. Dexmedetomidine, an $\alpha 2$-agonist available for ICU sedation, may reduce the duration of mechanical ventilation and enhance patient comfort

Objective To determine the efficacy of dexmedetomidine vs midazolam or propofol (preferred usual care) in maintaining sedation; reducing duration of mechanical ventilation; and improving patients' interaction with nursing care.

Design, Setting, and Patients Two phase 3 multicenter, randomized, double-blind trials carried out from 2007 to 2010. The MIDEX trial compared midazolam with dexmedetomidine in ICUs of 44 centers in 9 European countries; the PRODEX trial compared propofol with dexmedetomidine in 31 centers in 6 European countries and 2 centers in Russia. Included were adult ICU patients receiving mechanical ventilation who needed light to moderate sedation for more than 24 hours (midazolam, $\mathrm{n}=251$, vs dexmedetomidine, $\mathrm{n}=249$; propofol, $\mathrm{n}$ $=247$, vs dexmedetomidine, $\mathrm{n}=251$ ) .

Interventions Sedation with dexmedetomidine, midazolam, or propofol; daily sedation stops; and spontaneous breathing trials.

Main Outcome Measures For each trial, we tested whether dexmedetomidine was noninferior to control with respect to proportion of time at target sedation level (measured by Richmond Agitation-Sedation Scale) and superior to control with respect to duration of mechanical ventilation. Secondary end points were patients' ability to communicate pain (measured using a visual analogue scale [VAS]) and length of ICU stay. Time at target sedation was analyzed in per-protocol population (midazolam, $\mathrm{n}=233$, vs dexmedetomidine, $\mathrm{n}=227$; propofol, $\mathrm{n}=214$, vs dexmedetomidine, $\mathrm{n}=223$ ).

Results Dexmedetomidine/midazolam ratio in time at target sedation was 1.07 (95\% CI, 0.97-1.18) and dexmedetomidine/ propofol, 1.00 (95\% CI, 0.92-1.08). Median duration of mechanical ventilation appeared shorter with dexmedetomidine (123 hours [IQR, 67-337]) vs midazolam (164 hours [IQR, 92-380]; $P$ $=.03$ ) but not with dexmedetomidine (97 hours [IQR, 45-257]) vs propofol (118 hours [IQR, 48-327]; $P=.24$ ). Patients' interaction (measured using VAS) was improved with dexmedetomidine (estimated score difference vs midazolam, 19.7 [95\% CI, 15.2-24.2]; $P<.001$; and vs propofol, 11.2 [95\% CI, 6.4-15.9]; $P<.001)$. Length of ICU and hospital stay and mortality were similar. Dexmedetomidine vs midazolam patients had more hypotension (51/247 [20.6\%] vs 29/250 [11.6\%]; $P=.007)$ and bradycardia $(35 / 247[14.2 \%]$ vs $13 / 250[5.2 \%] ; P<.001)$.
Conclusions Among ICU patients receiving prolonged mechanical ventilation, dexmedetomidine was not inferior to midazolam and propofol in maintaining light to moderate sedation. Dexmedetomidine reduced duration of mechanical ventilation compared with midazolam and improved patients' ability to communicate pain compared with midazolam and propofol. More adverse effects were associated with dexmedetomidine.

1) Comments: This is the first large-scale study comparing dexmedetomidine with propofol in long-term sedation. In the PRODEX trial, dexmedetomidine has a tendency to reduce the ventilator days with a lower incidence of delirium. In the MIDEX trial, dexmedetomidine reduces the ventilator days. Patients are more alert and communicable when dexmedetomidine was administered in both trials. Revised guidelines ${ }^{9}$ for the management of pain, agitation, and delirium in adult patients in the ICU suggest that sedation strategies using nonbenzodiazepine sedatives (either propofol or dexmedetomidine) may be preferred over sedation with benzodiazepines (either midazolam or lorazepam) to improve the clinical outcomes in mechanically ventilated adult ICU patients. On the other hand, it is important to remain cautious about the higher incidence of hypotension and bradycardia with dexmedetomidine than with midazolam but comparable with propofol.

\section{Weaning from Mechanical Ventilation}

1. Effect of pressure support vs unassisted breathing through a tracheostomy collar on weaning duration in patients requiring prolonged mechanical ventilation: a randomized trial. Jubran et al. ${ }^{10}$ JAMA 2013; 309:671-7

Importance Patients requiring prolonged mechanical ventilation ( $>21$ days) are commonly weaned at long-term acute care hospitals (LTACHs). The most effective method of weaning such patients has not been investigated.

Objective To compare weaning duration with pressure support vs unassisted breathing through a tracheostomy collar in patients transferred to an LTACH for weaning from prolonged ventilation.

Design, Setting, and Participants Between 2000 and 2010, a randomized study was conducted in tracheotomized patients transferred to a single LTACH for weaning from prolonged ventilation. Of 500 patients who underwent a 5-day screening procedure, 316 did not tolerate the procedure and were randomly assigned to receive weaning with pressure support $(\mathrm{n}=155)$ or a tracheostomy collar $(\mathrm{n}=161)$. Survival at 6 - and 12-month time points was also determined.

Main Outcome Measure Primary outcome was weaning duration. Secondary outcome was survival at 6 and 12 months after enrollment. 
Results Of 316 patients, 4 were withdrawn and not included in analysis. Of 152 patients in the pressure-support group, 68 (44.7\%) were weaned; 22 (14.5\%) died. Of 160 patients in the tracheostomy collar group, 85 (53.1\%) were weaned; 16 (10.0\%) died. Median weaning time was shorter with tracheostomy collar use (15 days; interquartile range [IQR], 8-25) than with pressure support (19 days; IQR, 12-31), $P=.004$. The hazard ratio (HR) for successful weaning rate was higher with tracheostomy collar use than with pressure support (HR, 1.43; 95\% CI, 1.031.98; $P=.033$ ) after adjusting for baseline clinical covariates. Use of the tracheostomy collar achieved faster weaning than did pressure support among patients who did not tolerate the screening procedure between 12 and 120 hours (HR, 3.33; 95\% CI, 1.44-7.70; $P=.005$ ), whereas weaning time was equivalent with the 2 methods in patients who did not tolerate the screening procedure within 0 to 12 hours. Mortality was equivalent in the pressure-support and tracheostomy collar groups at 6 months (55.92\% vs $51.25 \%$; $4.67 \%$ difference, $95 \% \mathrm{CI},-6.4 \%$ to $15.7 \%$ ) and at 12 months ( $66.45 \%$ vs $60.00 \% ; 6.45 \%$ difference, $95 \% \mathrm{CI},-4.2 \%$ to $17.1 \%)$.

1) Comments: In the results, the factors associated with the successful weaning from prolonged mechanical ventilation are age, ventilator duration before randomization, frequencyto-tidal volume ratio, maximal inspiratory pressure, and weaning method. On the other hand, the weaning method is associated with the weaning duration only in the late failure group (screening failure between 12 and 120 hours). Of the 500 patients enrolled, $32 \%$ passed the initial tracheostomy collar challenge and could have been weaned before randomization. The results do not allow a simple expansion to patients in the ICU, because the study was conducted at a long-term acute care hospital. Nevertheless, patients who received weeks of mechanical ventilation may be less 'ventilator-dependent' and 'unassisted breathing with the tracheostomy collar' challenge may help to wean the patients earlier.

\section{Natriuretic peptide-driven fluid management during} ventilator weaning: a randomized controlled trial. Mekontso Dessap et al. ${ }^{11}$ Am J Respir Crit Care Med 2012;186:1256-63

Rationale: Difficult weaning from mechanical ventilation is often associated with fluid overload. B-type natriuretic peptide (BNP) has been proposed as a tool for predicting and detecting weaning failure of cardiovascular origin.

Objectives: To investigate whether fluid management guided by daily BNP plasma concentrations improves weaning outcomes compared with empirical therapy dictated by clinical acumen.

Methods: In a randomized controlled multicenter study, we allocated 304 patients to either a BNP-driven or physician-driven strategy of fluid management during ventilator weaning.... The primary end point was time to successful extubation. Measurements and Main Results: In the BNP-driven group, furosemide and acetazolamide were given more often and in higher doses than in the control group, resulting in a more negative median (interquartile range) fluid balance during weaning $(-2,320[-4,735,738]$ vs. $-180[-2,556,2,832] \mathrm{ml} ; P<0.0001)$. Time to successful extubation was significantly shorter with the BNP-driven strategy $(58.6[23.3,139.8]$ vs. $42.4[20.8,107.5]$ h; $P=0.034)$. The BNP-driven strategy increased the number of ventilator-free days but did not change length of stay or mortality. The effect on weaning time was strongest in patients with left ventricular systolic dysfunction. The two strategies did not differ significantly regarding electrolyte imbalance, renal failure, or shock.

Conclusions: Our results suggest that a BNP-driven fluid management strategy decreases the duration of weaning without increasing adverse events, especially in patients with left ventricular systolic dysfunction.

1) Comments: In the B-type natriuretic peptide (BNP)guided group, significantly fewer patients developed ventilator-associated pneumonia (9.2\% vs. $17.8 \%, \mathrm{p}=0.029)$. This appears to be due to the shorter time to extubation and the direct effect of the fluid balance on bacterial colonization and infectivity ${ }^{11}$. This study had several limitations. The study was unblinded and performed soon after the ARDSnet Fluid and Catheter Treatment Trial (FACTT) ${ }^{5}$. Therefore, the control group might not use the FACTT protocol. Nonetheless, this is the first trial of the BNP-guided fluid management strategy during weaning from mechanical ventilation. Measurement of BNP level is inexpensive and user-friendly, and helps guide the appropriate weaning from mechanical ventilation particularly for patients with left ventricular systolic dysfunction ${ }^{11,12}$. A longer ventilation time increases the risk of complications, such as pneumonia. Therefore, the time-to-event can be a clinically important primary endpoint in studies of sepsis or ARDS.

\section{Resuscitation Fluid in Sepsis}

1. Scandinavian starch for severe sepsis/septic shock (6S) trial

Hydroxyethyl starch 130/0.42 versus Ringer's acetate in severe sepsis. Perner et al. ${ }^{13}$ N Engl J Med 2012;367:12434

BACKGROUND Hydroxyethyl starch (HES) is widely used for fluid resuscitation in intensive care units (ICUs), but its safety and efficacy have not been established in patients with severe sepsis.

METHODS In this multicenter, parallel-group, blinded trial, we 
randomly assigned patients with severe sepsis to fluid resuscitation in the ICU with either 6\% HES 130/0.42 (Tetraspan) or Ringer's acetate at a dose of up to $33 \mathrm{ml}$ per kilogram of ideal body weight per day. The primary outcome measure was either death or end-stage kidney failure (dependence on dialysis) at 90 days after randomization.

RESULTS Of the 804 patients who underwent randomization, 798 were included in the modified intention-to-treat population. The two intervention groups had similar baseline characteristics. At 90 days after randomization, 201 of 398 patients (51\%) assigned to HES 130/0.42 had died, as compared with 172 of 400 patients (43\%) assigned to Ringer's acetate (relative risk, 1.17; 95\% confidence interval [CI], 1.01 to 1.36 ; $\mathrm{P}=0.03$ ); 1 patient in each group had end-stage kidney failure. In the 90day period, 87 patients (22\%) assigned to HES 130/0.42 were treated with renal-replacement therapy versus 65 patients (16\%) assigned to Ringer's acetate (relative risk, 1.35; 95\% CI, 1.01 to $1.80 ; \mathrm{P}=0.04$ ), and 38 patients ( $10 \%)$ and 25 patients $(6 \%)$, respectively, had severe bleeding (relative risk, 1.52; 95\% CI, 0.94 to 2.48; $\mathrm{P}=0.09$ ). The results were supported by multivariate analyses, with adjustment for known risk factors for death or acute kidney injury at baseline.

CONCLUSIONS Patients with severe sepsis assigned to fluid resuscitation with HES 130/0.42 had an increased risk of death at day 90 and were more likely to require renal-replacement therapy, as compared with those receiving Ringer's acetate.

1) Comments: The increased risk of death and the use of renal replacement therapy (RRT) with hydroxyethyl starch (HES) in the current study are similar to those from a previously published report ${ }^{14}$. In both trials ${ }^{13,14}$, the mortality started to increase around 20 days with HES, which might due to the late adverse effects of HES: a high fraction of HES is taken up and deposited in the tissues, where it cannot be metabolized and acts as a foreign body ${ }^{15}$. This can lead to impaired coagulation and increased use of red cells. The long-term toxic effect of HES appears to increase the number of deaths. Similarly, there is no benefit of colloids (modified gelatin, HES, and dextran) in resuscitation compared to crystalloid in metaanalysis $^{16}$. Moreover, HES produced higher mortality (pooled risk ratio, 1.10; 95\% CI, 1.02-1.19) compared to the other resuscitation fluids (crystalloid, albumin, and gelatin) after the 2002 Surviving Sepsis Campaign ${ }^{17}$.

This study demonstrates the certainty of harm with HES as a resuscitation fluid in severe sepsis.

\section{The crystalloid versus hydroxyethyl starch trial (CHEST)}

Hydroxyethyl starch or saline for fluid resuscitation in intensive care. Myburgh et al. ${ }^{18} \mathrm{~N}$ Engl J Med 2012; 367:1901-11

BACKGROUND The safety and efficacy of hydroxyethyl starch
(HES) for fluid resuscitation have not been fully evaluated, and adverse effects of HES on survival and renal function have been reported.

METHODS We randomly assigned 7000 patients who had been admitted to an intensive care unit (ICU) in a 1:1 ratio to receive either $6 \%$ HES with a molecular weight of $130 \mathrm{kD}$ and a molar substitution ratio of $0.4(130 / 0.4$, Voluven) in $0.9 \%$ sodium chloride or $0.9 \%$ sodium chloride (saline) for all fluid resuscitation until ICU discharge, death, or 90 days after randomization. The primary outcome was death within 90 days. Secondary outcomes included acute kidney injury and failure and treatment with renal-replacement therapy.

RESULTS A total of 597 of 3315 patients (18.0\%) in the HES group and 566 of 3336 (17.0\%) in the saline group died (relative risk in the HES group, 1.06; 95\% confidence interval [CI], 0.96 to $1.18 ; \mathrm{P}=0.26$ ). There was no significant difference in mortality in six predefined subgroups. Renal-replacement therapy was used in 235 of 3352 patients (7.0\%) in the HES group and 196 of 3375 (5.8\%) in the saline group (relative risk, 1.21 ; $95 \% \mathrm{CI}, 1.00$ to 1.45 ; $\mathrm{P}=0.04)$. In the HES and saline groups, renal injury occurred in $34.6 \%$ and $38.0 \%$ of patients, respectively $(\mathrm{P}=0.005)$, and renal failure occurred in $10.4 \%$ and $9.2 \%$ of patients, respectively $(\mathrm{P}=0.12)$. HES was associated with significantly more adverse events ( $5.3 \%$ vs. $2.8 \%, \mathrm{P}<0.001)$.

CONCLUSIONS In patients in the ICU, there was no significant difference in 90-day mortality between patients resuscitated with $6 \%$ HES $(130 / 0.4)$ or saline. However, more patients who received resuscitation with HES were treated with renalreplacement therapy.

1) Comments: Unlike the $6 \mathrm{~S}$ trial $^{13}$, this study did not find increased mortality with HES, compared to saline, for fluid resuscitation in the ICU. On the other hand, HES is associated with the increased risk of acute renal injury and the use of RRT, which are similar to previous results ${ }^{13,14,16,17}$. Based on the results of these trials ${ }^{13,14,16-18}$, the revised 2012 Surviving Sepsis Campaign Guideline ${ }^{19}$ recommends the use of crystalloids, not the use of HES, as the initial fluid of choice in the resuscitation of severe sepsis and septic shock. Therefore, the selection of a resuscitation fluid in the ICU requires scrupulous consideration of its safety and efficacy.

\section{Intensivist Staffing and Mortality in ICU}

1. Nighttime intensivist staffing and mortality among critically ill patients. Wallace et al. ${ }^{20} \mathrm{~N}$ Engl J Med 2012;366:2093-101

BACKGROUND Hospitals are increasingly adopting 24-hour intensivist physician staffing as a strategy to improve intensive care unit (ICU) outcomes. However, the degree to which nighttime intensivists are associated with improvements in the qual- 
ity of ICU care is unknown.

METHODS We conducted a retrospective cohort study involving ICUs that participated in the Acute Physiology and Chronic Health Evaluation (APACHE) clinical information system from 2009 through 2010, linking a survey of ICU staffing practices with patient-level outcomes data from adult ICU admissions. Multivariate models were used to assess the relationship between nighttime intensivist staffing and in-hospital mortality among ICU patients, with adjustment for daytime intensivist staffing, severity of illness, and case mix. We conducted a confirmatory analysis in a second, population-based cohort of hospitals in Pennsylvania from which less detailed data were available.

RESULTS The analysis with the use of the APACHE database included 65,752 patients admitted to 49 ICUs in 25 hospitals. In ICUs with low-intensity daytime staffing, nighttime intensivist staffing was associated with a reduction in risk-adjusted inhospital mortality (adjusted odds ratio for death, 0.62; $\mathrm{P}=0.04$ ). Among ICUs with high-intensity daytime staffing, nighttime intensivist staffing conferred no benefit with respect to riskadjusted in-hospital mortality (odds ratio, 1.08; $\mathrm{P}=0.78$ ). In the verification cohort, there was a similar relationship among daytime staffing, nighttime staffing, and in-hospital mortality. The interaction between nighttime staffing and daytime staffing was not significant $(\mathrm{P}=0.18)$, yet the direction of the findings were similar to those in the APACHE cohort.

CONCLUSIONS The addition of nighttime intensivist staffing to a low-intensity daytime staffing model was associated with reduced mortality. However, a reduction in mortality was not seen in ICUs with high-intensity daytime staffing.

1) Comments: Ideally, 24-hour intensivist staffing seems to improve ICU outcomes but it incurs a higher investment cost and leads to intensivists' burnout. This study presents no benefit of nighttime intensivist in-hospital mortality. In particular, nighttime intensivist staffing does not reduce mortality in the ICUs with high-intensity daytime staffing. However, the use of an alternative definition of nighttime staffing, which includes a nighttime intensivist or resident physician, is associated with reduced in-hospital mortality in both low-intensity ICUs (odds ratio, $0.42 ; 95 \% \mathrm{CI}, 0.29-0.59 ; \mathrm{p}<0.01)$ and high-intensity ICUs (odds ratio, 0.47; 95\% CI, 0.349-0.65; $\mathrm{p}<0.01$ ) $^{20}$.

Intensivists are a scarce resource, and the feasibility of 24-hour intensivist coverage is questionable. For nighttime staffing performance, it is important to consider its costeffectiveness and intensivists' exhaustion. To improve the ICU outcomes, first of all, what do you think about primary transfer of care to the intensivist or mandatory consultation with the intensivist in the daytime? Most importantly, the staffing practice should be individualized according to the each circumstance of the ICU, and efforts to improve the quality of critical care should be made.

\section{Other Notable Articles}

Lung protective mechanical ventilation is associated with a substantial long-term survival benefit for patients with ALI over a two-year follow-up ${ }^{21}$, and is associated with a lower incidence of ALI, death, pulmonary infection, and lung collapse in patients without ARDS $^{22}$. Early deep sedation within 48 hours of commencing ventilation is an independent negative predictor of the time to extubation, hospital death, and 180day mortality ${ }^{23}$. Among adult patients with severe sepsis, treatment with combined meropenem and moxifloxacin compared with meropenem alone does not result in less organ failure $^{24}$. Intensive glucose control (81 to $108 \mathrm{mg} / \mathrm{dL}$ ) leads to moderate and severe hypoglycemia, both of which are associated with an increased risk of death ${ }^{25}$.

\section{Summary}

These most recent articles show remarkable findings: there is no benefit of HFOV in ARDS; there is no benefit of HES for fluid resuscitation in the ICU; neuropsychological impairment is increased in ARDS survivors with fluid restriction; BNPguided weaning, or unassisted breathing with the tracheostomy collar shortens the time to extubation; adding daily sedation interruption to protocolized sedation does not shorten time to extubation; dexmedetomidine is feasible for sedation during mechanical ventilation; and nighttime intensivist staffing has no benefit in ICUs with high-intensity staffing. These findings highlight the need for further studies into developing critical care.

\section{References}

1. Ferguson ND, Cook DJ, Guyatt GH, Mehta S, Hand L, Austin $\mathrm{P}$, et al. High-frequency oscillation in early acute respiratory distress syndrome. N Engl J Med 2013;368:795-805.

2. Young D, Lamb SE, Shah S, MacKenzie I, Tunnicliffe W, Lall R, et al. High-frequency oscillation for acute respiratory distress syndrome. N Engl J Med 2013;368:806-13.

3. Sud S, Sud M, Friedrich JO, Meade MO, Ferguson ND, Wunsch $\mathrm{H}$, et al. High frequency oscillation in patients with acute lung injury and acute respiratory distress syndrome (ARDS): systematic review and meta-analysis. BMJ 2010;340:c2327.

4. Mikkelsen ME, Christie JD, Lanken PN, Biester RC, Thompson BT, Bellamy SL, et al. The adult respiratory distress syndrome cognitive outcomes study: long-term neuropsychological function in survivors of acute lung injury. Am J Respir Crit Care Med 2012;185:1307-15.

5. National Heart, Lung, and Blood Institute Acute Respiratory Distress Syndrome (ARDS) Clinical Trials Network, Wiedemann HP, Wheeler AP, Bernard GR, Thompson BT, Hayden 
D, et al. Comparison of two fluid-management strategies in acute lung injury. N Engl J Med 2006;354:2564-75.

6. Mehta S, Burry L, Cook D, Fergusson D, Steinberg M, Granton J, et al. Daily sedation interruption in mechanically ventilated critically ill patients cared for with a sedation protocol: a randomized controlled trial. JAMA 2012;308:1985-92.

7. Girard TD, Kress JP, Fuchs BD, Thomason JW, Schweickert WD, Pun BT, et al. Efficacy and safety of a paired sedation and ventilator weaning protocol for mechanically ventilated patients in intensive care (Awakening and Breathing Controlled trial): a randomised controlled trial. Lancet 2008;371:126-34.

8. Jakob SM, Ruokonen E, Grounds RM, Sarapohja T, Garratt C, Pocock SJ, et al. Dexmedetomidine vs midazolam or propofol for sedation during prolonged mechanical ventilation: two randomized controlled trials. JAMA 2012;307:1151-60.

9. Barr J, Fraser GL, Puntillo K, Ely EW, Gelinas C, Dasta JF, et al. Clinical practice guidelines for the management of pain, agitation, and delirium in adult patients in the intensive care unit. Crit Care Med 2013;41:263-306.

10. Jubran A, Grant BJ, Duffner LA, Collins EG, Lanuza DM, Hoffman LA, et al. Effect of pressure support vs unassisted breathing through a tracheostomy collar on weaning duration in patients requiring prolonged mechanical ventilation: a randomized trial. JAMA 2013;309:671-7.

11. Mekontso Dessap A, Roche-Campo F, Kouatchet A, Tomicic V, Beduneau G, Sonneville R, et al. Natriuretic peptide-driven fluid management during ventilator weaning: a randomized controlled trial. Am J Respir Crit Care Med 2012;186:1256-63.

12. Zapata L, Vera P, Roglan A, Gich I, Ordonez-Llanos J, Betbese AJ. B-type natriuretic peptides for prediction and diagnosis of weaning failure from cardiac origin. Intensive Care Med 2011;37:477-85.

13. Perner A, Haase N, Guttormsen AB, Tenhunen J, Klemenzson G, Aneman A, et al. Hydroxyethyl starch 130/0.42 versus Ringer's acetate in severe sepsis. N Engl J Med 2012;367:12434.

14. Brunkhorst FM, Engel C, Bloos F, Meier-Hellmann A, Ragaller $\mathrm{M}$, Weiler $\mathrm{N}$, et al. Intensive insulin therapy and pentastarch resuscitation in severe sepsis. N Engl J Med 2008;358:125-39.

15. Bellmann R, Feistritzer C, Wiedermann CJ. Effect of molecular weight and substitution on tissue uptake of hydroxyethyl starch: a meta-analysis of clinical studies. Clin Pharmacokinet
2012;51:225-36.

16. Perel P, Roberts I. Colloids versus crystalloids for fluid resuscitation in critically ill patients. Cochrane Database Syst Rev 2012;6:CD000567.

17. Zarychanski R, Abou-Setta AM, Turgeon AF, Houston BL, McIntyre L, Marshall JC, et al. Association of hydroxyethyl starch administration with mortality and acute kidney injury in critically ill patients requiring volume resuscitation: a systematic review and meta-analysis. JAMA 2013;309:678-88.

18. Myburgh JA, Finfer S, Bellomo R, Billot L, Cass A, Gattas D, et al. Hydroxyethyl starch or saline for fluid resuscitation in intensive care. N Engl J Med 2012;367:1901-11.

19. Dellinger RP, Levy MM, Rhodes A, Annane D, Gerlach H, Opal SM, et al. Surviving sepsis campaign: international guidelines for management of severe sepsis and septic shock: 2012. Crit Care Med 2013;41:580-637.

20. Wallace DJ, Angus DC, Barnato AE, Kramer AA, Kahn JM. Nighttime intensivist staffing and mortality among critically ill patients. N Engl J Med 2012;366:2093-101.

21. Needham DM, Colantuoni E, Mendez-Tellez PA, Dinglas VD, Sevransky JE, Dennison Himmelfarb CR, et al. Lung protective mechanical ventilation and two year survival in patients with acute lung injury: prospective cohort study. BMJ 2012;344:e2124.

22. Serpa Neto A, Cardoso SO, Manetta JA, Pereira VG, Esposito DC, Pasqualucci Mde O, et al. Association between use of lung-protective ventilation with lower tidal volumes and clinical outcomes among patients without acute respiratory distress syndrome: a meta-analysis. JAMA 2012;308:1651-9.

23. Shehabi Y, Bellomo R, Reade MC, Bailey M, Bass F, Howe B, et al. Early intensive care sedation predicts long-term mortality in ventilated critically ill patients. Am J Respir Crit Care Med 2012;186:724-31.

24. Brunkhorst FM, Oppert M, Marx G, Bloos F, Ludewig K, Putensen C, et al. Effect of empirical treatment with moxifloxacin and meropenem vs meropenem on sepsis-related organ dysfunction in patients with severe sepsis: a randomized trial. JAMA 2012;307:2390-9.

25. NICE-SUGAR Study Investigators, Finfer S, Liu B, Chittock DR, Norton R, Myburgh JA, et al. Hypoglycemia and risk of death in critically ill patients. N Engl J Med 2012;367:1108-18. 